\title{
MiR-29b suppresses peritoneal metastases through inhibition of the mesothelial-mesenchymal transition (MMT) of human peritoneal mesothelial cells
}

\author{
Yuki Kimura \\ Jichi Medical University \\ Hideyuki Ohzawa \\ Jichi Medical University \\ Hideyo Miyato \\ Jichi Medical University \\ Yuki Kaneko \\ Jichi Medical University \\ Kazuya Takahashi \\ Jichi Medical University \\ Mineyuki Tojo \\ Jichi Medical University \\ Hironori Yamaguchi \\ Jichi Medical University \\ Kentaro Kurashina \\ Jichi Medical University \\ Shin Saito \\ Jichi Medical University \\ Yoshinori Hosoya \\ Jichi Medical University \\ Alan Kawarai Lefor \\ Jichi Medical University \\ Naohiro Sata \\ Jichi Medical University \\ Joji Kitayama ( $\nabla$ kitayama@jichi.ac.jp ) \\ Jichi Medical University \\ Akira Saito \\ Jichi Medical University
}




\section{Research Article}

Keywords: miR-29, mesothelial mesenchymal transition, peritoneal metastasis, fibronectin

Posted Date: August 4th, 2021

DOl: https://doi.org/10.21203/rs.3.rs-774286/v1

License: (c) (i) This work is licensed under a Creative Commons Attribution 4.0 International License. Read Full License 


\section{Abstract}

Background: Peritoneal dissemination is a major metastatic pathway for gastrointestinal and ovarian malignancies. The miR-29b family is downregulated in peritoneal fluids in patients with peritoneal metastases (PM). We examined the effect of miR-29b on mesothelial cells (MC) which play critical a role in the development of PM through mesothelial-mesenchymal transition (MMT). Methods: Human peritoneal mesothelial cells (HPMCs) were isolated from surgically resected omental tissue and MMT induced by stimulation with $10 \mathrm{ng} / \mathrm{ml}$ TGF-b1. MiR-29b mimics and negative control miR were transfected by lipofection using RNAiMAX and the effects on the MMT evaluated in vitro. Results: HPMC produced substantial amounts of miR-29b which was markedly inhibited by TGF-b1. TGF-b1 stimulation of HPMC induced morphological changes with decreased expression of E-cadherin and calretinin, and increased expression of vimentin and fibronectin. TGF-b1 also enhanced proliferation and migration of HPMC as well as adhesion of tumor cells in a fibronectin dependent manner. However, all events were strongly abrogated by simultaneous transfection of miR-29b. Conclusion: MiR-29b inhibits TGF-b1 induced MMT and replacement of miR-29b in the peritoneal cavity might be effective to prevent development of PM partly through the effects on MC.

\section{Introduction}

The peritoneum is a well-known metastatic site for gastrointestinal and ovarian malignancies ${ }^{1,2}$. Peritoneal carcinomatosis often develops which is associated with an extremely poor prognosis ${ }^{3-5}$. Although, peritoneal metastases (PM) are likely to develop from free intraperitoneal tumor cells exfoliated from the serosal surface of a primary tumor ${ }^{6,7}$, the mechanisms underlying the process leading to the development of PM have not been fully elucidated.

The peritoneum is composed of a single layer of flat mesothelial cells (MC) and a thin layer of submesothelial connective tissue. An intact MC layer is believed to act as the first barrier against bacterial invasion and tumor attachment. However, after mechanical damage or peritoneal dialysis, MC lose their intercellular junctions, and increase their migratory capacity, producing a large amount of extracellular matrix components and a wide range of inflammatory, profibrotic and angiogenic factors, causing postsurgical peritoneal adhesions ${ }^{8}$ or peritoneal fibrosis ${ }^{9,10}$. This phenomenon, named as mesothelialmesenchymal transition (MMT), has been shown to be a critical step in the process of the development of $\mathrm{PM}{ }^{11-14}$.

MicroRNAs (miRNAs) are a class of small noncoding RNA molecules, 20-23 nucleotides in length, that regulate post-transcription gene expression by interfering with the translation of multiple target mRNAs and play important roles in the regulation of a variety of cell functions. In the miR-29 family, their function and biological significance have been extensively analyzed ${ }^{15,16} 17$. In oncology, miR-29s have been shown to induce cell cycle arrest and apoptosis of tumor cells ${ }^{18}$, suppress epithelial mesenchymal transition (EMT) ${ }^{19,20}$, and inhibit angiogenesis ${ }^{19,21,22}$, suggesting that miR-29s predominantly function 
as tumor suppressor. In fact, miR-29 is reported to be downregulated in various tumors as compared with normal counterparts ${ }^{23}$. We consistently found that the miR-29 family in exosomes derived from peritoneal fluid was markedly downregulated in patients with peritoneal metastases from gastric cancer

24. However, the role of miR-29s in the development of PM is not fully understood. In this study, we evaluated the effects of miR-29b on the cellular function of MC to examine its possible roles in the development of PM.

\section{Results}

\section{Expression of miR-29b in HPMC was decreased by TGF- $\beta 1$}

HPMC were isolated from omental tissue obtained from patients who underwent sleeve gastrectomy and cultured in DMEM supplemented with 15\% FBS. Digital PCR analysis revealed that HPMC produced substantial levels of miR-29b, similar to gastric cancer cell, MKN45 and peripheral blood mononuclear cells (PBMC) but less than less than that by mesenchymal stem cells (Fig.1A). However, when HPMC were cultured with $10 \mathrm{ng} / \mathrm{ml}$ TGF- $\beta 1$ for 24 hours, the expression of miR29b was markedly decreased (Fig.1B).

\section{Transfection of miR-29b mimic suppresses phenotypic changes of HPMC by TGF- $\beta 1$}

After $48 \mathrm{hr}$-culture with TGF- $\beta 1$, HPMCs changed their morphology from round to spindle shape (Fig.2). Stimulation with TGF- $\beta 1$ significantly downregulated the expression of E-cadherin and calretinin, while markedly enhancing vimentin expression. However, lipofection of miR-29b mimics, but not with negative control-miR, significantly suppressed the morphological changes with reduced expression of vimentin and restored the expression of E-cadherin and calretinin induced by TGF- $\beta 1$ (Fig.2).

\subsection{Transfection of miR-29b mimic suppresses the proliferation and migration of HPMCs}

The proliferation of HPMC was not significantly altered by TGF- $\beta$. However, transfection with miR$29 \mathrm{~b}$ slightly decreased proliferation $(n=3, p<0.05)$ (Fig.3A). Although HPMC rarely migrated to the lower surface of culture inserts, they did migrate to $10 \%$ FCS after stimulation with 10ng/ml TGF- $\beta 1$ (12.0 \pm 7.2 vs $70.5 \pm 12.2$ counts/HPF, $n=3, p<0.001)$. When pretreated with miR29b mimic, the number of migrated cells was greatly reduced compared with negative control miRNA ( $3.4 \pm 1.3$ vs $17.9 \pm 3.1$ counts/HPF, $n=3$, p $<0.001$ ) (Figs. 3 B,C).

\section{Transfection of miR-29b mimic in HPMC suppresses tumor cell adhesion mediated by the RGD/b1 integrin cascade}

In adhesion experiments, a few NUGC-4 and MKN45 attached to the HPMC monolayer. However, after stimulation of HPMC with TGF- $\beta 1$, number of NUGC-4 and MKN45 attached to the HPMC markedly increased (NUGC-4, 6.7 \pm 2.3 vs $50.7 \pm 22.0$ counts/HPF, $n=5, p<0.001$; MKN45; $13.7 \pm 3.7$ vs $147.9 \pm 43.4$ counts/HPF, $n=5, p<0.0001$ ) (Figs. 4 A,B). However, enhanced adhesion of NUGC-4 and MKN45 was 
significantly reduced by the presence of RGD peptide (NUGC-4; $23.9 \pm 5.9$ counts/HPF, $n=5, p<0.0001$, MKN45; $6.2 \pm 2.7$ counts/HPF, $n=5, p<0.0001$ ) or anti- $\beta 1$ integrin $m A b$ (NUGC-4; $16.3 \pm 6.1$ counts/HPF, $n=5$, $p<0.0001 ;$ MKN45; $12.3 \pm 5.4$ counts/HPF, $n=5, p<0.0001$ ). (Figs. $4 A, B)$.

When HPMC were transfected with miR-29b, but not with negative control, the enhancing effects of TGF-b1 on the adhesion of NUGC-4 and MKN45 were totally abrogated (NUGC-4;18.0 \pm 5.2 vs $300.0 \pm 119.8$ counts/HPF, $n=5, p<0.001 ;$ MKN45, 7.5 \pm 2.4 vs $219.9 \pm 51.7$ counts/HPF, $n=5, p<0.0001$ ) (Figs $4 C, D)$

\section{Transfection of miR-29b mimic to HPMC suppresses the expression of fibronectin (FN)}

Since adhesion data suggest a contribution of fibronectin (FN) in this adhesion cascade, we next examined the effect on the expression of FN on HPMC. FN was faintly detected on the surface of resting HPMC by immunohistochemistry analysis. However, after stimulation with TGF- $\beta 1$, HPMC strongly expressed FN (Fig.5). When miR-29b mimics were transfected with TGF- $\beta 1$ stimulation, the expression of FN was not increased (Fig.5).

\section{Discussion}

MC cover the inner surface of the peritoneal cavity and secrete large amounts of lubricant that facilitates intracoelomic movement and actively contributes to fluid transport, coagulation, fibrinolysis and local immunity ${ }^{25}$. MC are initially considered to be a mechanical barrier to tumor metastases in the peritoneal space ${ }^{26,27}$. However, recent studies have suggested that MC activated by TGF-b acquire a mesenchymallike phenotype through the MMT which mediate attachment and enhance the migration of disseminated tumor cells to the sub-peritoneal space like cancer associated fibroblasts (CAFs) ${ }^{28-30}$.

In this study, we confirmed that HPMC acquire the phenotype of CAF with enhanced proliferative and migratory properties by TGF-b1 stimulation. However, all of the observed phenomena were largely abrogated by the transfection of miR-29b. Extensive studies have already shown that the miR-29 family regulates multiple oncogenic processes, including epigenetics, proteostasis, metabolism, proliferation, apoptosis, metastasis, fibrosis, angiogenesis, and immunomodulation ${ }^{15} 17$. In particular, miR-29 strongly suppresses the epithelial mesenchymal transition (EMT) of tumor cells ${ }^{19,31}$ through targeting the expression of various genes including catenin, b1-integrin and matrix metalloproteinases (MMPs) ${ }^{15} 17$. Given that these EMT-related genes are largely shared with MC, the results of this study indicate that TGFb1 induces MMT mainly through the downregulation of miR-29b.

Treatment with TGF-b1 markedly upregulated FN expression on an HPMC monolayer and induced robust adhesion of gastric cancer cells which was inhibited by an antibody to b1 integrin and RGD peptide. This is consistent with the results in previous studies showing that FN-b1 integrin is an important adhesion pathway between MC and tumor cell ${ }^{30,32}$. However, the enhanced adhesion by TGFb1 was also abrogated by transfection of miR-29b. The miR-29 family has been shown to suppress the synthesis of extracellular matrix proteins such as collagens, laminin and fibrillin in embryonic 
fibroblasts ${ }^{33}$, trabecular meshwork cells ${ }^{34}$ and hepatic stellate cells ${ }^{35}$. Zhu et al showed that miR-29b downregulates FN production via Hsp47 in breast cancer cells ${ }^{36}$. The results of the present study are consistent with those results and suggest that transduction of miR-29b in MC effectively inhibits the attachment of disseminated tumor cells on MC which is the initial step in the development of PM.

In previous studies, we found that the expression of exosomal miR-29b in peritoneal fluid was markedly reduced in patients who developed peritoneal recurrence after curative resection of gastric cancer ${ }^{37}$ as well as patients with PM ${ }^{38}$. Generally, expression of miR-29 in tumor cells decreased mainly via hypermethylation of the $\mathrm{CpG}$ areas of the miR-29 promoter lesion ${ }^{39,40}$, and the diminished expression of miR-29 is believed to promote tumor progression ${ }^{16,17}$. However, since the number of disseminated tumor cells is thought to be low during the initial step of the development of PM, it is unlikely that reduced miR-29b in peritoneal fluids is dependent on tumor cells.

In this study, we found that resting HPMC produce substantial amount of miR-29b which was markedly suppressed by the addition of TGF-b1. This is consistent with previous studies showing that the TGF-b-SMAD signal suppresses miR-29 expression 41,42 and considerable levels of TGF-b could be detected in malignant effusions ${ }^{43,44}$. These facts suggest that reduced production of miR-29b in MC downregulates the amount of miR-29b in the abdominal cavity which is a prerequisite for the development of PM. Taken together, this suggests that replacement of miR-29b in peritoneal space using appropriate delivery methods may be effective to prevent PM partly through its effects on MC.

\section{Methods}

\section{Reagents}

The oligonucleotides of miR-29b-3p mimics and negative control miRNA were synthesized by Thermo Fisher Scientific (Waltham, MA). The sequence of the oligonucleotides used for miR-29b-3pmimics

5'-UAGCACCAUUUGAAAUCAGUGUU-3.

Lipofectamine RNAiMAX was purchased from Invitrogen (Carlsbad, CA). Rabbit mAbs to E-cadherin and calretinin, were purchased from Abcam (Cambridge, UK), Cell Signaling Technology (Danvers, MA). Recombinant- TGF-b1 was purchased from R\&D systems (Minneapolis, MN). Rabbit anti-vimentin mAb and anti-rabbit Ig conjugated with AlexaFluor 488 or AlexaFluor $595 \AA$ were from Invitrogen. DAPI was obtained from Dojindo (Kumamoto, Japan). Anti-integrin b1 mAb and RGD peptide were purchased from Cayman Chemical Co. (Ann Arbor, MI).

\section{Cell culture}

Human gastric cancer cells, NUGC-4, MKN45 were obtained from Riken (Tsukuba JAPAN), and a bone marrow derived mesenchymal stem cell line, UE6E7T-12, was obtained from the Japan Health Science Foundation (Tokyo, Japan). The cells were cultured in Dulbecco's Modified Eagle Medium 
(DMEM) supplemented with 10\% fetal bovine serum (FBS; Sigma, St. Louis, MO), $100 \mathrm{U} / \mathrm{mL}$ penicillin, and $100 \mathrm{mg} / \mathrm{mL}$ streptomycin (Life Technologies, Grand Island, NY) at $37^{\circ} \mathrm{C}$ in a $5 \% \mathrm{CO} 2$ cell culture incubator.

\section{Isolation and culture of human peritoneal mesothelial cells (HPMC)}

HPMC were isolated from 2-4 cm3 samples of omentum collected from consenting patients undergoing sleeve gastrectomy. Omental samples were momentarily placed in a half TrypLE Express (Thermo Fisher Scientific, Waltham, MA) with pure PBS, and incubated in a thermostatic tank at $37^{\circ} \mathrm{C}$ for $2 \mathrm{~h}$. The supernatants were collected after filtration by $100 \mu \mathrm{m}$-pore nylon mesh, were centrifuged at 1,500 rpm and $4^{\circ} \mathrm{C}$ for 5 minites. Explants were seeded into collagen coated $10 \mathrm{~cm} 2$ tissue culture dishes (Falcon, Becton Dickinson, Oxford, UK) and cultured in DMEM with 20\% FBS, $100 \mathrm{U} / \mathrm{mL}$ penicillin, and $100 \mathrm{mg} / \mathrm{mL}$ streptomycin at $37^{\circ} \mathrm{C}$ in $5 \% \mathrm{CO} 2$ cell culture incubator (Riera, McCulloch et al. 2006).

\section{Sample collection and miRNA purification and digital polymerase reaction (PCR)}

Cell line miRNAs were isolated from cell pellets using the MiRNeasy kit (Qiagen, Hilden, Germany), according to the manufactures' instructions. cDNA was synthesized starting from $100 \mu \mathrm{g}$ of extracted RNA using the TaqMan miRNA Reverse Transcription Kit and miRNA-specific stem-loop primers (Applied BioSystems, Foster City, CA) following manufacturer's instructions. Total miR-29b-3p cell levels were then quantified using the digital (dd) PCR system (Bio-Rad Laboratories, Hercules, CA). Briefly, $10 \mu \mathrm{g}$ of synthesized cDNA were added to a $20 \mu$ PCR reaction mixture containing $10 \mu$ l of digital PCR ${ }^{\text {TM }}$ supermix (Bio-Rad Laboratories), $1 \mu$ l of TaqMan primer/probe mix (Applied BioSystems) and RNase-free H2O. Droplets were generated by loading the mixture onto a plastic cartridge with $70 \mu$ l of QX100 Droplet Generation oil (Bio-Rad Laboratories). Cartridges were then placed into the QX200 Droplet Generator (BioRad Laboratories). The droplets generated from each sample were transferred to a 96-well PCR plate (Eppendorf, Hamburg, Germany), and PCR amplification was carried out on the C1000 Touch Thermal Cycler (Bio-Rad Laboratories), according to the manufacturer's protocol. The plate was then loaded on the QX200 Droplet Reader (Bio-Rad Laboratories) and read automatically. The fraction of PCR-positive droplets was quantified assuming a Poisson distribution. QuantaSoft software was used to obtain the concentration results in number of copies per microliter for each sample.

\section{Introduction of MMT and transfection of miR-29b-3p}

HPMCs were seeded in 6-well plates at 50\%-60\% confluence and induced with $10 \mathrm{ng} / \mathrm{ml}$ of TGF$\beta 1$. Then, the cells were transfected with miR-29b-3p mimic or negative controls with lipofectamine RNAiMAX in HPMC at a final concentration of $50 \mathrm{nM}$, and cultured for $48 \mathrm{hr}$ at $37^{\circ} \mathrm{C}$, according to the manufacturer's instructions and then used for the following experiments

\section{Immunofluorescence}


HPMC $\left(5 \times 10^{4}\right)$ were plated in 24-well collagen coated plates, incubated with $10 \mathrm{ng} / \mathrm{ml}$ of TGF-b1 and then transfected miR-29b or negative controls for $48 \mathrm{~h}$. Cells were washed with PBS, fixed in $4 \%$ paraformaldehyde for $10 \mathrm{~min}$ at $37^{\circ} \mathrm{C}$ and then permeabilized with $0.5 \%$ Tween-20 in PBS for $20 \mathrm{~min}$. Subsequently, cells were blocked for $1 \mathrm{~h}$ with $3 \%$ BSA in PBS at room temperature. Cells were then incubated for $1 \mathrm{hr}$ at room temperature with mAbs to E-cadherin (1:200), calretinin (1:500), vimentin (1:1000), and fibronectin (FN) (1:150). Then, cells were washed 3 times with PBS and incubated for $30 \mathrm{~min}$ at room temperature with the appropriate fluorescence conjugated secondary anti-rabbit antibodies conjugated with AlexaFluor 488 or AlexaFluor $595 \AA$ (1:2000). Lastly, the nucleus was counterstained with DAPI (1:1000) for $5 \mathrm{~min}$. Glass coverslips were placed on slides and the preparations were visualized under a fluorescence microscope (Keyense, Osaka, JAPAN).

\section{Cell proliferation}

HPMCs (1.0x104 cells) transfected with miR-29b or negative controls were cultured with or without TGF-b1 in 96-well culture plate for $24 \mathrm{hr}$ and incubated with MTS (DOJINDO, Kumamoto, Japan) diluted in normal culture media at $37^{\circ} \mathrm{C}$ for an additional $3 \mathrm{hr}$. Proliferation rates were determined and quantification was performed on a microtiter plate reader (Spectra Rainbow; Tecan) according to the manufacturer's protocol.

\section{Cell migration}

HPMCs (5.0x105 cells) transfected with miR-29b or negative controls were cultured with or without TGF-b1 for 48 hours. Cells were re-suspended in DMEM and seeded on an 8- $\mu \mathrm{m}$ pore membrane in 24well plate and 10\% FBS was added to the lower chamber as a chemoattractant. After $24 \mathrm{~h}$, non-migrated cells were gently removed with a cotton swab. Migrated cells on the lower surface of the culture insets were stained with Dif-Quick (Sysmex, Kobe, Japan) and counted.

\section{Cell adhesion assay}

HPMCs (5.0x104 cells) transfected with miR-29b or negative controls were cultured with or without TGF-b1 in 24-well culture plates. Fluorescein labelled NUGC-4 (1.0 x104 cells/well) were then added, incubated for $15 \mathrm{~min}$ for all cells to attach to the MC monolayer. After gentle washing with warmed media 3 times, the number of NUGC cells remaining attached were counted under a fluorescent microscope.

\section{Statistical analysis}

Data were represented as mean \pm standard deviation. The significance of the differences between groups was assessed with a one-way ANOVA using GraphPad Prism8. Differences were considered significant when $\mathrm{P}<0.05$.

\section{References}


1. Colombo, N. et al. Ovarian cancer. Crit Rev Oncol Hematol, 60, 159-179 https://doi.org/10.1016/j.critrevonc.2006.03.004 (2006).

2. Soucisse, M. L., Liauw, W., Hicks, G. \& Morris, D. L. Early postoperative intraperitoneal chemotherapy for lower gastrointestinal neoplasms with peritoneal metastasis: a systematic review and critical analysis. Pleura Peritoneum, 4, 20190007 https://doi.org/10.1515/pp-2019-0007 (2019).

3. Yan, T. D., Black, D., Savady, R. \& Sugarbaker, P. H. Systematic review on the efficacy of cytoreductive surgery combined with perioperative intraperitoneal chemotherapy for peritoneal carcinomatosis from colorectal carcinoma. J Clin Oncol, 24, 4011-4019 https://doi.org/10.1200/JC0.2006.07.1142 (2006).

4. Yang, X. J., Li, Y. \& Yonemura, Y. Cytoreductive surgery plus hyperthermic intraperitoneal chemotherapy to treat gastric cancer with ascites and/or peritoneal carcinomatosis: Results from a Chinese center. J Surg Oncol, 101, 457-464 https://doi.org/10.1002/jso.21519 (2010).

5. Kitayama, J. Intraperitoneal chemotherapy against peritoneal carcinomatosis: Current status and future perspective. Surg Oncol, https://doi.org/10.1016/j.suronc.2014.03.004 (2014).

6. Koga, S. et al. Prognostic significance of intraperitoneal free cancer cells in gastric cancer patients. $J$ Cancer Res Clin Oncol, 108, 236-238 (1984).

7. Sodek, K. L., Murphy, K. J., Brown, T. J. \& Ringuette, M. J. Cell-cell and cell-matrix dynamics in intraperitoneal cancer metastasis. Cancer Metastasis Rev, 31, 397-414 https://doi.org/10.1007/s10555-012-9351-2 (2012).

8. Sandoval, P. et al. Mesothelial-to-mesenchymal transition in the pathogenesis of post-surgical peritoneal adhesions. J Pathol, 239, 48-59 https://doi.org/10.1002/path.4695 (2016).

9. Yanez-Mo, M. et al. Peritoneal dialysis and epithelial-to-mesenchymal transition of mesothelial cells. N Engl J Med, 348, 403-413 https://doi.org/10.1056/NEJMoa020809 (2003).

10. Lopez-Cabrera, M. Mesenchymal Conversion of Mesothelial Cells Is a Key Event in the Pathophysiology of the Peritoneum during Peritoneal Dialysis. Adv Med 2014, 473134, doi:10.1155/2014/473134 (2014).

11. Rynne-Vidal, A., Jimenez-Heffernan, J. A., Fernandez-Chacon, C., Lopez-Cabrera, M. \& Sandoval, P. The Mesothelial Origin of Carcinoma Associated-Fibroblasts in Peritoneal Metastasis. Cancers (Basel), 7, 1994-2011 https://doi.org/10.3390/cancers7040872 (2015).

12. Saito, H. et al. Importance of human peritoneal mesothelial cells in the progression, fibrosis, and control of gastric cancer: inhibition of growth and fibrosis by tranilast., 21, 55-67 https://doi.org/10.1007/s10120-017-0726-5 (2018).

13. Sandoval, P. et al. Carcinoma-associated fibroblasts derive from mesothelial cells via mesothelial-tomesenchymal transition in peritoneal metastasis. J Pathol, 231, 517-531 https://doi.org/10.1002/path.4281 (2013).

14. Gordillo, C. H. et al. Mesothelial-to-Mesenchymal Transition Contributes to the Generation of Carcinoma-Associated Fibroblasts in Locally Advanced Primary Colorectal Carcinomas. Cancers (Basel), 12, https://doi.org/10.3390/cancers12020499 (2020). 
15. Kwon, J. J., Factora, T. D., Dey, S. \& Kota, J. A. Systematic Review of miR-29 in Cancer. Mol Ther Oncolytics, 12, 173-194 https://doi.org/10.1016/j.omto.2018.12.011 (2019).

16. Jiang, H., Zhang, G., Wu, J. H. \& Jiang, C. P. Diverse roles of miR-29 in cancer (review). Oncol Rep, 31, 1509-1516 https://doi.org/10.3892/or.2014.3036 (2014).

17. Alizadeh, M. et al. The potential role of miR-29 in health and cancer diagnosis, prognosis, and therapy. J Cell Physiol, 234, 19280-19297 https://doi.org/10.1002/jcp.28607 (2019).

18. Garzon, R. et al. MicroRNA 29b functions in acute myeloid leukemia., 114, 5331-5341 https://doi.org/10.1182/blood-2009-03-211938 (2009).

19. Chou, J. et al. GATA3 suppresses metastasis and modulates the tumour microenvironment by regulating microRNA-29b expression. Nat Cell Biol, 15, 201-213 https://doi.org/10.1038/ncb2672 (2013).

20. Wang, Y. et al. Chemotherapy-Induced miRNA-29c/Catenin-delta Signaling Suppresses Metastasis in Gastric Cancer. Cancer Res, 75, 1332-1344 https://doi.org/10.1158/0008-5472.CAN-14-0787 (2015).

21. Yang, Z. et al. MiR-29a modulates the angiogenic properties of human endothelial cells. Biochem Biophys Res Commun, 434, 143-149 https://doi.org/10.1016/j.bbrc.2013.03.054 (2013).

22. Simons, M., Gordon, E. \& Claesson-Welsh, L. Mechanisms and regulation of endothelial VEGF receptor signalling. Nat Rev Mol Cell Biol, 17, 611-625 https://doi.org/10.1038/nrm.2016.87 (2016).

23. Jacobsen, A. et al. Analysis of microRNA-target interactions across diverse cancer types. Nat Struct Mol Biol, 20, 1325-1332 https://doi.org/10.1038/nsmb.2678 (2013).

24. Ohzawa, H. et al. Exosomal microRNA in peritoneal fluid as a biomarker of peritoneal metastases from gastric cancer. Ann Gastroenterol Surg, 4, 84-93 https://doi.org/10.1002/ags3.12296 (2020).

25. Mutsaers, S. E. The mesothelial cell. Int J Biochem Cell Biol, 36, 9-16 (2004).

26. Iwanicki, M. P. et al. Ovarian cancer spheroids use myosin-generated force to clear the mesothelium. Cancer Discov, 1, 144-157 https://doi.org/10.1158/2159-8274.CD-11-0010 (2011).

27. Niedbala, M. J., Crickard, K. \& Bernacki, R. J. Interactions of human ovarian tumor cells with human mesothelial cells grown on extracellular matrix. An in vitro model system for studying tumor cell adhesion and invasion. Exp Cell Res, 160, 499-513 https://doi.org/10.1016/0014-4827(85)90197-1 (1985).

28. Kojima, M. et al. Human subperitoneal fibroblast and cancer cell interaction creates microenvironment that enhances tumor progression and metastasis. PLoS One, 9, e88018 https://doi.org/10.1371/journal.pone.0088018 (2014).

29. Sun, F., Feng, M. \& Guan, W. Mechanisms of peritoneal dissemination in gastric cancer. Oncol Lett, 14, 6991-6998 https://doi.org/10.3892/ol.2017.7149 (2017).

30. Yoshihara, M. et al. Ovarian cancer-associated mesothelial cells induce acquired platinum-resistance in peritoneal metastasis via the FN1/Akt signaling pathway. Int J Cancer, 146, 2268-2280 https://doi.org/10.1002/ijc.32854 (2020). 
31. Wong, C. C. et al. Lysyl oxidase-like 2 is critical to tumor microenvironment and metastatic niche formation in hepatocellular carcinoma., 60, 1645-1658 https://doi.org/10.1002/hep.27320 (2014).

32. Kenny, H. A. et al. Mesothelial cells promote early ovarian cancer metastasis through fibronectin secretion. J Clin Invest, 124, 4614-4628 https://doi.org/10.1172/JCI74778 (2014).

33. Yang, T. et al. miR-29 mediates TGFbeta1-induced extracellular matrix synthesis through activation of PI3K-AKT pathway in human lung fibroblasts. J Cell Biochem, 114, 1336-1342 https://doi.org/10.1002/jcb.24474 (2013).

34. Luna, C., Li, G., Qiu, J., Epstein, D. L. \& Gonzalez, P. Role of miR-29b on the regulation of the extracellular matrix in human trabecular meshwork cells under chronic oxidative stress. Mol Vis, 15, 2488-2497 (2009).

35. Kwiecinski, M. et al. Hepatocyte growth factor (HGF) inhibits collagen I and IV synthesis in hepatic stellate cells by miRNA-29 induction. PLoS One, 6, e24568 https://doi.org/10.1371/journal.pone.0024568 (2011).

36. Zhu, J. et al. Chaperone Hsp47 Drives Malignant Growth and Invasion by Modulating an ECM Gene Network. Cancer Res, 75, 1580-1591 https://doi.org/10.1158/0008-5472.CAN-14-1027 (2015).

37. Ohzawa, H. et al. Reduced expression of exosomal miR29s in peritoneal fluid is a useful predictor of peritoneal recurrence after curative resection of gastric cancer with serosal involvement. Oncol Rep, 43, 1081-1088 https://doi.org/10.3892/or.2020.7505 (2020).

38. Ohzawa, $\mathrm{H}$. et al. Exosomal microRNA in peritoneal fluid as a biomarker of peritoneal metastases from gastric cancer.. Annals of Gastrointestinal Surgery In press(2019).

39. Li, Y. et al. Epigenetic deregulation of miR-29a and miR-1256 by isoflavone contributes to the inhibition of prostate cancer cell growth and invasion. Epigenetics, 7, 940-949 https://doi.org/10.4161/epi.21236 (2012).

40. Cui, H. et al. Deregulation between miR-29b/c and DNMT3A is associated with epigenetic silencing of the $\mathrm{CDH} 1$ gene, affecting cell migration and invasion in gastric cancer. PLoS One, 10, e0123926 https://doi.org/10.1371/journal.pone.0123926 (2015).

41. Wang, B. et al. Suppression of microRNA-29 expression by TGF-beta1 promotes collagen expression and renal fibrosis. J Am Soc Nephrol, 23, 252-265 https://doi.org/10.1681/ASN.2011010055 (2012).

42. Harmanci, D., Erkan, E. P., Kocak, A. \& Akdogan, G. G. Role of the microRNA-29 family in fibrotic skin diseases. Biomed Rep, 6, 599-604 https://doi.org/10.3892/br.2017.900 (2017).

43. Ikubo, A. et al. A possible role of TGF-beta in the formation of malignant effusions. Clin Immunol Immunopathol, 77, 27-32 https://doi.org/10.1016/0090-1229(95)90133-7 (1995).

44. Yigit, R. et al. Cytokine analysis as a tool to understand tumour-host interaction in ovarian cancer. Eur J Cancer, 47, 1883-1889 https://doi.org/10.1016/j.ejca.2011.03.026 (2011).

\section{Declarations}

Acknowledgments 
This work was supported by the Japan Society for the Promotion of Science (20K07704). We thank We thank Ms N. Nishiaki, J. Shinohara, H. Hatakeyama, N. Nishiaki and I. Nieda for technical and clerical work.

\section{Author Contributions}

Y.K., H.O., H.M. and J.K. conceived and designed the experiments and performed the experiments. Y.K., A.S., M.T. K.K., S.S., H.Y., and Y.S. provided the omental samples and performed experiments. A.K.L, N.S, and J.K wrote the main manuscript. All authors reviewed the manuscript.

\section{Additional Information}

All authors declare that they have no competing financial interests.

\section{Figures}

(A)

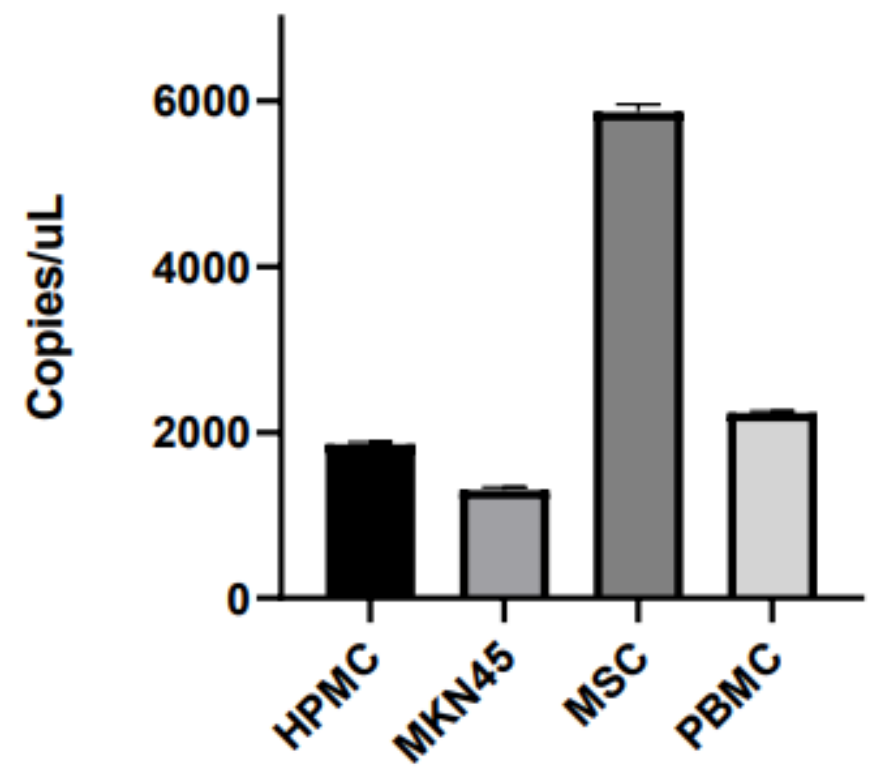

(B)

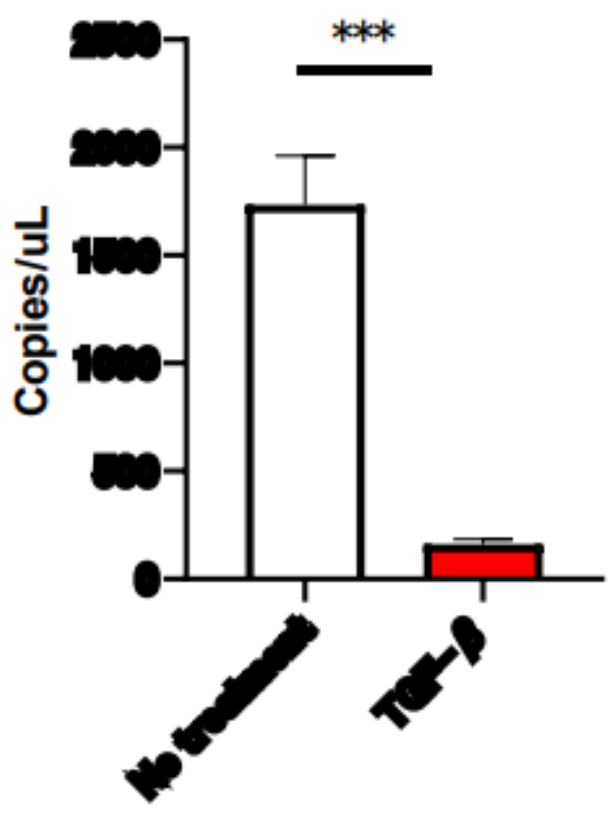

Figure 1

A: Expression of miR-29b in cell lines and human peritoneal mesothelial cells (HPMC). Total miR-29b was quantified using the digital droplet polymerase chain reaction (dd-PCR) system. B. HPMC were cultured in 
the presence of $10 \mathrm{ng} / \mathrm{ml}$ TGF- $囚 1$ or without was quantified with dd-PCR. $* \star * ; p<0.001$.

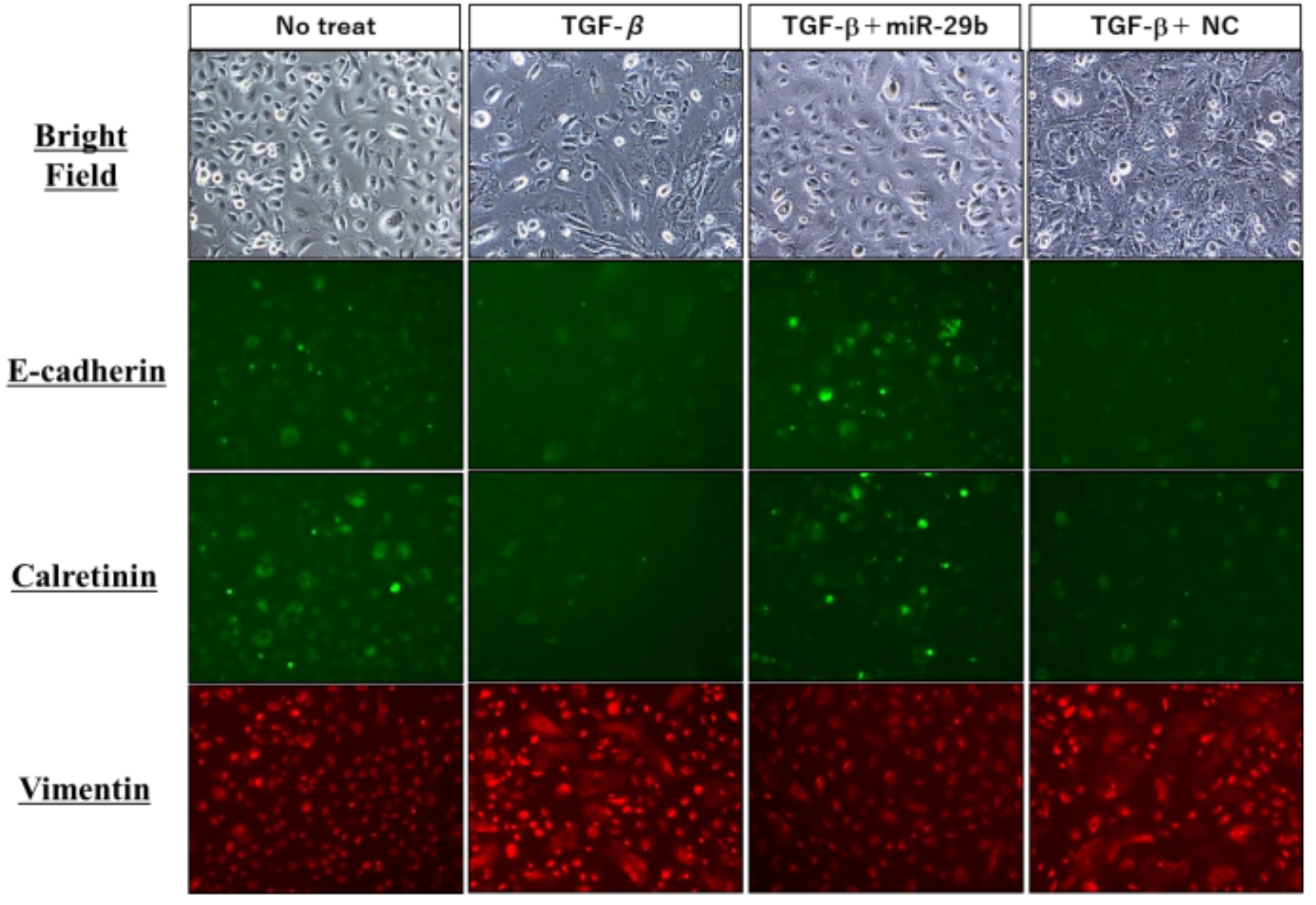

Figure 2

Phenotypic changes of human peritoneal mesothelial cells (HPMC) by stimulation with TGF- $\beta 1$. HPMC were cultured with 10ng/ml TGF- $\beta 1$ for 48 hours. In some wells, HPMC were transfected with miR-29b-3p mimic or negative control with lipofectamine RNAiMAX at a final concentration of 50nM, and cultured with TGF- $\beta 1$ for $48 \mathrm{hr}$ at $37^{\circ} \mathrm{C}$. Morphological changes by light microscopy and expression of E-cadherin, calretinin and vimentin were observed with fluorescein microscopy. Magnification: 400x. 


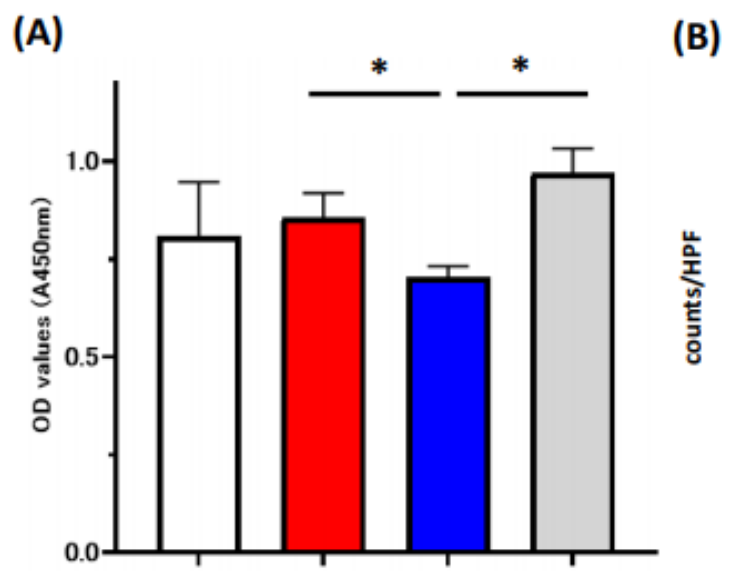

(C)

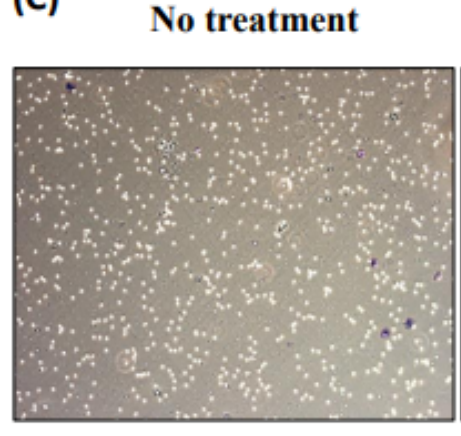

(B)

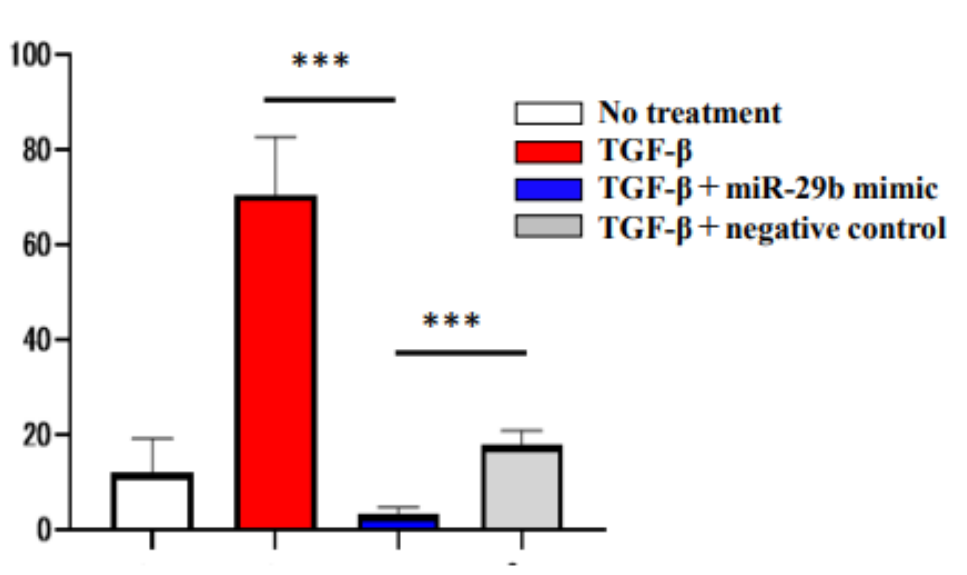

TGF- $\beta+$ miR-29b

Figure 3

MiR-29b inhibits proliferation and migration of human peritoneal mesothelial cells (HPMC). (A) HPMC were cultured as described in the legend of Figure 2 and proliferation for 3 hours was determined by the MTT assay. Data show mean \pm standard deviation in 1 of the 3 different experiments. (B) HPMC were resuspended in DMEM and seeded on $8-\mu \mathrm{m}$ pore membrane in 24 -well plate and $10 \%$ FBS was added to the lower chamber as a chemoattractant and the number of cells that migrated to the lower surface of culture insets were counted. Data show mean \pm standard deviation in 1 of the 3 different experiments. $; p<0.05$, $\star \star * ; p<0.001$. 
(A) NUGC-4

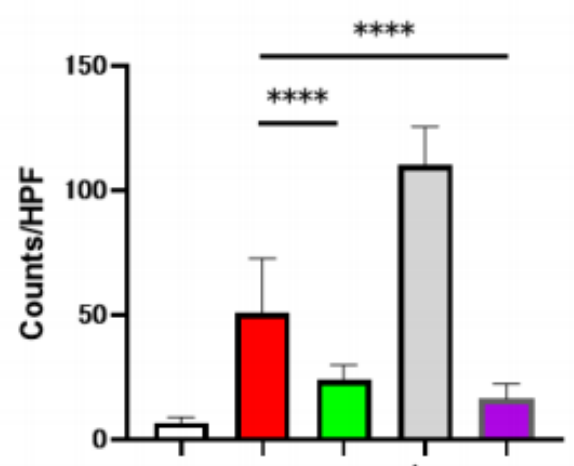

(C) NUGC-4

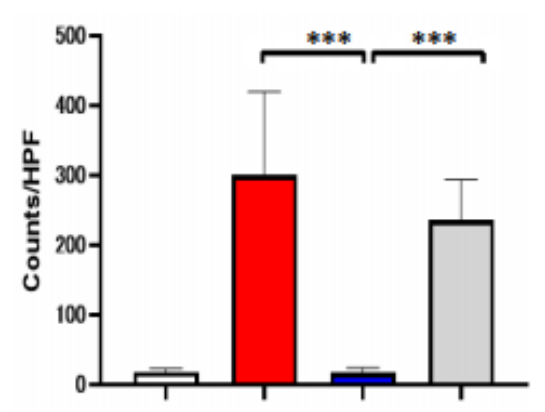

(B) MKN45

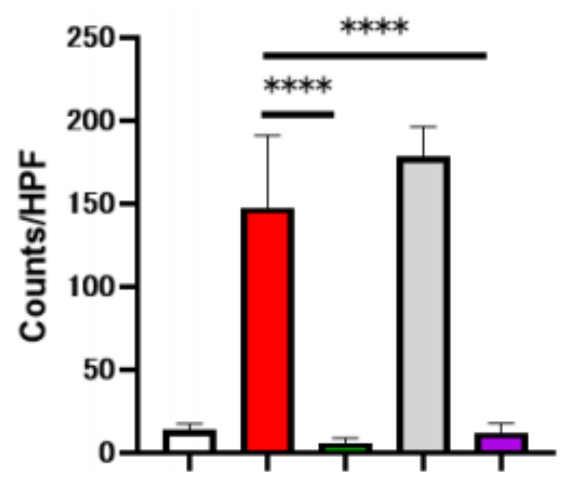

(D) MKN45

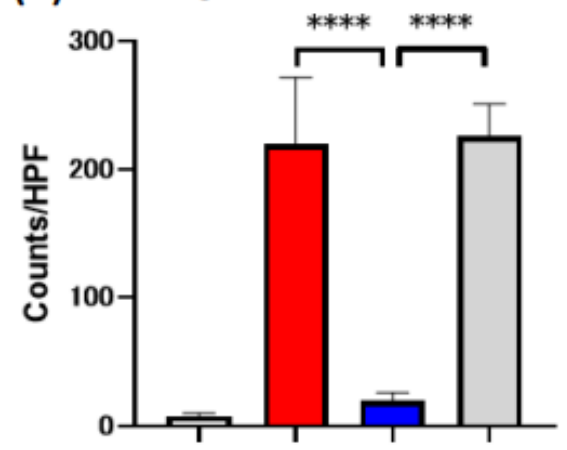

No treat

TGF- $\beta$

TGF- $\beta+$ RGD peptide

TGF- $\beta$ + isotype control

TGF- $\beta$ + anti- $\beta 1$ integrin

\section{Figure 4}

Adhesion of gastric cancer cells on human peritoneal mesothelial cells (HPMC). HPMC were cultured with TGF-Ruas described in the legend of Figure 2 and then fluorescein labelled NUGC-4 and MKN45 were added, incubated for $15 \mathrm{~min}$ on the mesothelial cell (MC) monolayer. After gentle washing with warmed media for 3 times, the number of NUGC-4 cells remaining attached were counted under a fluorescent

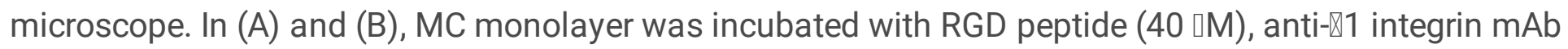
or isotype control mouse lgG $(20 \mathrm{gg} / \mathrm{ml})$ before the addition of tumor cells. In (C) and (D), MC monolayer was pretreated with miR-29b mimic or negative control as described in the legend of Figure 2. Data show mean \pm standard deviation in 1 of the 2 different experiments $* \star \star ; p<0.001, \star \star \star *: p<0.0001$. 


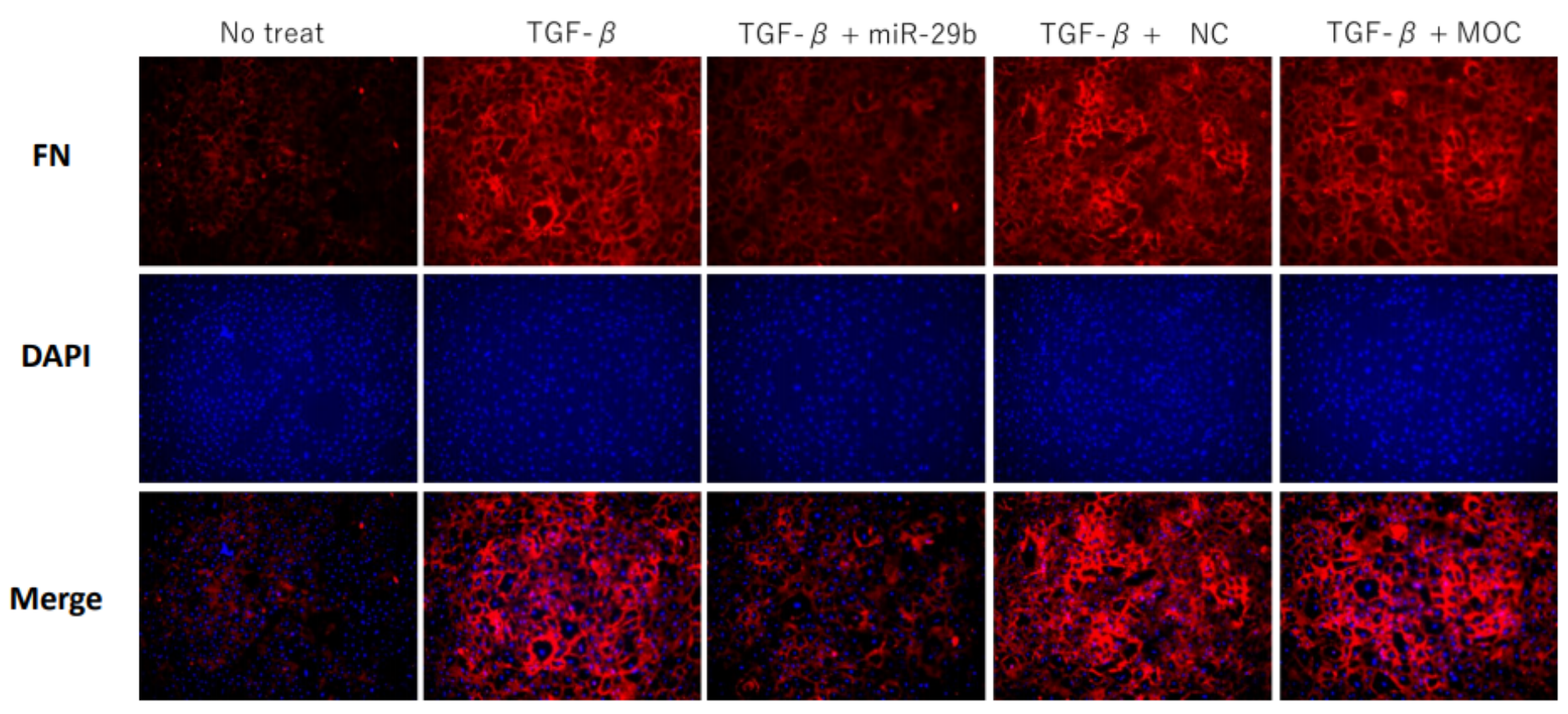

\section{Figure 5}

Expression of fibronectin (FN) on human peritoneal mesothelial cells (HPMC). HPMC were cultured as described in the legend of Figure 2, immunostained with DAPI and anti-FN mAb and surface expression of FN examined with fluorescein microscopy. Red; FN; Blue, nuclear deoxyribonucleic acid (DNA). Magnification: 400x. 